\title{
Quantized Oversampled Filter Banks with Erasures*
}

\author{
Pier Luigi Dragotti ${ }^{1} \quad$ Jelena Kovačević ${ }^{2} \quad$ Vivek K Goyal $^{2}$ \\ ${ }^{1}$ Audio-Visual Communications Laboratory \\ Swiss Federal Institute of Technology, Lausanne, Switzerland. \\ ${ }^{2}$ Mathematics of Communications Research \\ Bell Labs, Lucent Technologies. \\ dragotti@lcavsun1.epfl.ch, jelena@bell-labs.com,v.goyal@ieee.org.
}

\begin{abstract}
Oversampled filter banks can be used to enhance resilience to erasures in communication systems in much the same way that finite-dimensional frames have previously been applied. This paper extends previous finite dimensional treatments to frames and signals in $l_{2}(\mathcal{Z})$ with frame expansions that can be implemented efficiently with filter banks. It is shown that tight frames attain best performance. In particular, if encoding with a uniform frame, the quantization error is minimized if and only if the frame is tight. In case of one erasure and if encoding with a strongly uniform frame, tight frames are still optimal. In case of more erasures, an expression for the mean square error is given and some general considerations are presented.
\end{abstract}

\section{Introduction}

The use of frame expansions in signal processing has recently become quite popular, due to resilience to additive noise [4], resilience to quantization [6] and greater freedom to capture signal characteristics $[1,2]$. A frame gives a redundant representation of a signal and this redundancy can be exploited in different ways. The authors of $[7,8]$ have proposed to use this redundancy as a way to reduce the effect of losses in a packet-based communication system. That work deals with finite-dimensional frames and finite-dimensional signals.

In this paper we investigate the more general case of infinite-dimensional signals and oversampled filter banks which represent a convenient way to implement an important class of frames in $l_{2}(\mathcal{Z})[3,11]$. The encoding structure considered in this work is depicted in Figure 1: An input sequence $x[n]$ is fed through an $M$-channel FIR filter bank followed by downsampling by $N(N<M)$. The $M$ output sequences are then separately scalar quantized with uniform scalar quantizers and sent over $M$ different channels. Each channel either works perfectly or not at all. The decoder receives only $M-e$ of the quantized output sequences, where $e$ is the number of erasures during the transmission. We assume that there are no more than $M-N$ erasures. The reconstruction process is linear. We wish to find filter bank properties

${ }^{*}$ This work was performed while Pier Luigi Dragotti was a summer intern at Bell Labs. 
that minimize the mean square error (MSE) between the input and the reconstructed sequences.

To analyze cases with more than $M-N$ erasures requires a statistical model for the input sequence. In $[5,9,12]$, the input sequence is a stationary Gaussian source; in $[5,12]$ the case $M=N=2$ and one erasure is considered, while in [9] the case $M=3$ and $N=2$ and up to two erasures is analyzed. In this work we do not make any assumptions on the input source. However, we use a statistical model for the quantization error; the reconstruction then depends only on the characteristics of the filter bank.

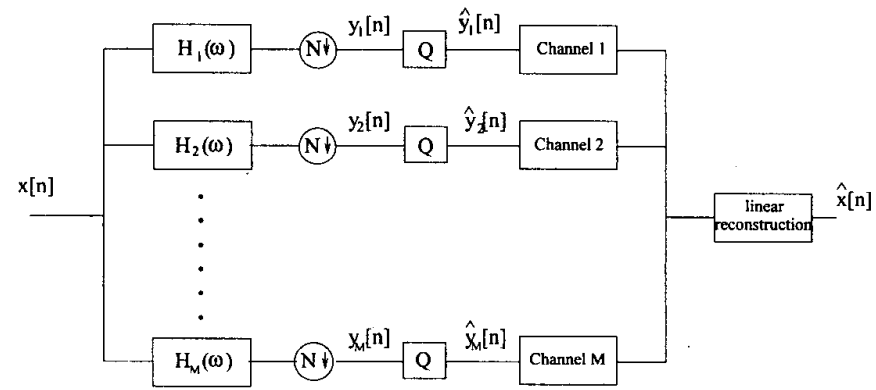

Figure 1: Abstraction of a communication system using an $M$-channel filter bank with downsampling by $N$.

The paper is organized as follows: Section 2 summarizes the main properties of frames and oversampled filter banks and establishes the notation. In Section 3 we show some examples of oversampled filter banks. In Sections 4 and 5 we analyze the effect of quantization and erasures on the MSE.

\section{Frame Expansions and Oversampled Filter Banks}

This review of frames and oversampled filter banks is based on [3, 4, 8]. For convenience we start with the finite-dimensional case, that is, we consider only frames in $\mathcal{C}^{N}$

A family of $M$ vectors $\Phi=\left\{\varphi_{k}\right\}_{k=1}^{M} \in \mathcal{C}^{N}$ constitutes a frame if for any vector $x \in \mathcal{C}^{N}$ there exist two constants $A>0$ and $B<\infty$ such that:

$$
A\|x\|^{2} \leq\|y\|^{2}=\sum_{k=1}^{M}\left|\left\langle x, \varphi_{k}\right\rangle\right|^{2} \leq B\|x\|^{2} .
$$

When $A=B$ the frame is tight. A frame is uniform if $\left\|\varphi_{k}\right\|=1$ for $k=1, \ldots, M$. We will use notation: TF for a tight frame, UF for a uniform frame and UTF for a uniform tight frame.

If we denote vectors as columns then the frame operator $F$ associated with $\Phi$ is an $M \times N$ matrix with the $i$ th row equal to $\varphi_{i}^{*}$. ${ }^{1}$ We then define an output vector

\footnotetext{
${ }^{1}$ The superscript ${ }^{*}$ denotes the Hermitian transpose.
} 
with $y=F x$. The properties of a frame can be conveniently defined using its frame operator $F$. For example, a frame is tight if and only if $F^{*} F=A I_{N}$, where $I_{N}$ is the $N \times N$ identity matrix while a UTF satisfies $F^{*} F=\frac{M}{N} I_{N}$. Vector $x$ can be reconstructed from $y$ using the so called pseudo-inverse:

$$
F^{\dagger}=\left(F^{*} F\right)^{-1} F^{*} .
$$

If we call $\lambda_{i}$ the eigenvalues of $F^{*} F$, then:

1. the sum of the eigenvalues of $F^{*} F$ equals the sum of the lengths of the frame vectors: $\sum_{i=1}^{N} \lambda_{i}=\operatorname{tr}\left(F^{*} F\right)=\operatorname{tr}\left(F F^{*}\right)=\sum_{k=1}^{M} \varphi_{k}^{*} \varphi_{k}=\sum_{k=1}^{M}\left\|\varphi_{k}\right\|^{2}$,

2. for a UF the sum of the eigenvalues equals $M$,

3. for a $\mathrm{TF}, F^{*} F$ has eigenvalue $A$ with multiplicity $N$,

4. for a UTF, $F^{*} F$ has eigenvalue $\frac{M}{N}$ with multiplicity $N$.

Consider again the filter bank shown in Figure 1. Call $H_{i}(\omega)=\left[H_{i 1}(\omega), H_{i 2}(\omega), \ldots H_{i N}(\omega)\right]^{*}$ the polyphase representation of the $i$ th analysis filter where

$$
H_{i j}(\omega)=\sum_{n=-\infty}^{\infty} h_{i}[n N-j] e^{-j n \omega} .
$$

Call $\mathbf{H}(\omega)$ the corresponding $M \times N$ polyphase analysis matrix, which is a matrix whose $i$ th row equals $H_{i}^{*}(\omega)$. Many properties can be stated easily in terms of this matrix:

Proposition 1 ([3]) A filter bank implements a frame decomposition in $l_{2}(\mathcal{Z})$ if and only if its polyphase analysis matrix is of full rank on the unit circle.

Proposition 2 ([3]) A filter bank implements a tight frame expansion in $l_{2}(\mathcal{Z})$ if and only if $\mathbf{H}^{*}(\omega) \mathbf{H}(\omega)=A I_{N}$.

Proposition 3 ([10]) An $M \times N$ polyphase matrix $\mathbf{H}(\omega)$ represents a tight frame if and only if it has the following decomposition: $\mathbf{H}(\omega)=\mathrm{U}(\omega) F$, where $\mathrm{U}(\omega)$ is an $M \times M$ paraunitary matrix $\left(\mathbf{U}^{*}(\omega) \mathbf{U}(\omega)=I_{M}\right)$ and $F$ is an $M \times N$ matrix such that. $F^{*} F=A I_{N}$.

Moreover, any paraunitary matrix can be decomposed into a sequence of elementary matrices such as rotations and delays $[10]$.

The pseudo-inverse is defined as in the finite-dimensional case:

$$
\mathbf{H}^{\dagger}(\omega)=\left(\mathbf{H}^{*}(\omega) \mathbf{H}(\omega)\right)^{-1} \mathbf{H}^{*}(\omega)
$$

A frame implemented with filter banks is uniform if: $\left\|h_{i}[n]\right\|=1, \quad i=1, \ldots, M$ or, using Parseval's relation, if: $\frac{1}{2 \pi} \int_{-\pi}^{\pi} \sum_{j=1}^{N}\left|H_{i j}(\omega)\right|^{2} d \omega=1, \quad i=1, \ldots, M$. We now introduce a new definition:

Definition 1 (Strongly uniform frame) A frame expansion in $l_{2}(\mathcal{Z})$ implemented by an $M \times N$ polyphase matrix $\mathbf{H}(\omega)$ is strongly uniform if: 


$$
\sum_{j=1}^{N}\left|H_{i j}(\omega)\right|^{2}=1, \quad i=1, \ldots, M
$$

In other words, strongly uniform frame is implemented by a filter bank which is uniform for each fixed $\omega$. Clearly, strongly uniform frames are a subset of uniform frames. However, notice that if $F$ is a frame operator corresponding to a uniform frame in $\mathcal{C}^{N}$ and if we assume $\mathbf{H}(\omega) \doteq F$ then the resulting oversampled filter bank is strongly uniform. Moreover, notice that a square paraunitary matrix is automatically strongly uniform. Further examples of strongly uniform frames will be shown in the next section.

If we call $\lambda_{i}(\omega)$ the spectral eigenvalues of $\mathbf{H}^{*}(\omega) \mathbf{H}(\omega)$, then:

1. the integral sum of the spectral eigenvalues of $\mathbf{H}^{*}(\omega) \mathbf{H}(\omega)$ equals the sum of the filters' norms: $\frac{1}{2 \pi} \int_{-\pi}^{\pi} \sum_{i=1}^{N} \lambda_{i}(\omega) d \omega=\sum_{i=1}^{M}\left\|h_{i}[n]\right\|^{2}$;

2. for a UF, the integral sum of the eigenvalues equals $M$;

3. for a $\mathrm{TF}, \mathbf{H}^{*}(\omega) \mathbf{H}(\omega)$ has eigenvalues constant over the unit circle and equal to $A$ with multiplicity $N: \lambda_{i}(\omega)=A, \quad i=1, \ldots, N$;

4. for a UTF, $\mathbf{H}^{*}(\omega) \mathbf{H}(\omega)$ has eigenvalues constant over the unit circle and equal to $\frac{M}{N}$ with multiplicity $N$.

\section{Examples of Uniform and Strongly Uniform Frames}

Oversampled filter banks are sometimes preferred to classical critically downsampled filter banks for their greater design freedom. However, this freedom makes the actual design difficult.

One of the most used families of oversampled filter banks is the nondownsampled one. It is obtained by eliminating the downsampling in the filter bank scheme. If the analysis and synthesis filters are power complementary (i.e. the synthesis filters are the time reversed versions of the analysis ones) then the corresponding frame is tight and uniform but not strongly uniform.

It will be shown in next sections that strongly uniform tight frames constitute an important class of frames. We propose the following factorization to design polyphase matrices corresponding to strongly uniform tight frames:

$$
\mathbf{H}(\omega)=F \mathbf{U}(\omega)
$$

where $F$ is a uniform tight frame in $\mathcal{C}^{N}$ and $\mathrm{U}(\omega)$ is an $N \times N$ paraunitary matrix. It is easy to see that such a polyphase matrix corresponds to a strongly uniform tight frame.

Note the difference between this factorization and the one in Proposition $3(\mathbf{H}(\omega)=$ $\mathbf{U}(\omega) F)$. The order of the elements is reversed, so in this last factorization, the paraunitary matrix has size $M \times M$, while in our factorization it has size $N \times N(N<M)$. This is not surprising since the family of polyphase matrices with the factorization $\mathbf{H}(\omega)=\mathbf{U}(\omega) F$ represents the more general class of tight frames and not the restricted class of strongly uniform tight frames. 
Although we cannot claim that our factorization includes all possible strongly uniform tight frames, we can state the following:

Theorem 1 Define an equivalence relation by bundling a frame (implemented with an FIR oversampled filter bank) with all frames that result from rigid rotations of its elements as well as negation or shift of some individual ones (i.e. $h_{i}[n] \rightarrow-h_{i}[n-k]$ $k \in \mathcal{Z}$ ). When $M=N+1$, there is a single equivalence class for all strongly uniform tight frames.

Proof: See Appendix A.1.

Since a UTF $F$ in $\mathcal{C}^{N}$. can be seen as a strongly uniform tight frame in $l_{2}(\mathcal{Z})$ (i.e. $\mathbf{H}(\omega)=F)$, Theorem 1 basically says that the factorization in (2) essentially includes all the possible strongly uniform tight frames when $M=N+1$ (up to a shift or negation of some individual elements).

\section{Quantized Oversampled Filter Banks}

In this section we will analyze the effect of quantization on the performance of the system. For the moment we assume that there are no erasures during transmission. We want the reconstruction operator to be linear, that is, we want it to be implemented by a synthesis filter bank. The reconstruction operator that we will use is the pseudo-inverse (1).

We will assume that the quantization error can be treated as additive white noise with variance $\sigma^{2}=\Delta^{2} / 12$, where $\Delta$ represents the step size of the quantizer and each quantizer has the same step size. We further assume that the noisy sequences generated by two different channels are independent. This can be expressed as:

$$
\hat{y}_{i}[n]=y_{i}[n]+w_{i}[n]
$$

and

$$
E\left[w_{i}[n] w_{j}^{*}[n-m]\right]=\sigma^{2} \delta_{i j} \delta[m] .
$$

Under this assumption (input sequences corrupted by additive noise), the pseudoinverse is the best linear reconstruction operator in the mean square sense [4]. Moreover, it can be shown that the MSE due to quantization is:

$$
M S E=\frac{1}{2 \pi} \int_{-\pi}^{\pi} \frac{\sigma^{2}}{N} \sum_{i=1}^{N} \frac{1}{\lambda_{i}(\omega)} d \omega,
$$

where $\lambda_{i}(\omega), \quad i=1, . ., N$ denote the spectral eigenvalues of $\mathbf{H}^{*}(\omega) \mathbf{H}(\omega)$. Recall that the integral sum of the eigenvalues is constant and if we are encoding with a uniform frame, it is equal to $M$. Thus, we want to minimize the MSE given the constraint that the integral sum of the eigenvalues is constant. This occurs when the eigenvalues are equal and constant over $\omega$ which is true if and only if the original frame is tight. We can then state the following theorem: ${ }^{2}$

${ }^{2}$ Proofs of Theorems 2 and 5 and derivation of (6) are omitted due to the lack of space. 
Theorem 2 When encoding with a filter bank implementing a uniform frame and decoding with the pseudo-inverse under the noise model (3)-(4), the MSE is minimum if and only if the frame is tight. Then:

$$
M S E_{0}=\frac{N}{M} \sigma^{2}
$$

\section{$5 \quad$ Introducing Erasures}

Here we consider the effect of erasures on the structure of the frame and on the MSE. We denote by $E$ the index set of erasures and by $\mathbf{H}_{E}(\omega)$ the polyphase matrix after $e=|E|$ erasures. $\mathbf{H}_{E}(\omega)$ is an $(M-e) \times N$ matrix obtained by deleting the $E$ numbered rows from the $M \times N$ polyphase matrix $\mathbf{H}(\omega)$. The first question to be answered is under which conditions $\mathbf{H}_{E}(\omega)$ still represents a frame. We then study the effect of erasures on the MSE.

\subsection{Effect of Erasures on the Structure of a Frame}

Our aim is to use the pseudo-inverse operator to reconstruct after $e$ erasures. The pseudo-inverse matrix is defined only if the matrix $\mathbf{H}_{E}(\omega)$ is still a frame, that is, if and only if it is still of full rank on the unit circle. This leads to the following definition:

Definition 2 An oversampled filter bank which implements a frame expansion represented by a polyphase matrix $\mathbf{H}(\omega)$ is said to be robust to $e=|E|$ erasures if and only if for any index set $E$ of erasures, $\mathbf{H}_{\mathrm{E}}(\omega)$ is of full rank on the unit circle.

Let us consider first the case where there is only one erasure.

Theorem 3 An oversampled filter bank which implements a uniform tight frame is robust to one erasure if and only if

$$
\sum_{j=1}^{N}\left|H_{i j}(\omega)\right|^{2}<\frac{M}{N} \text { for all } i=1, \ldots, M, \text { for all } \omega .
$$

Proof: See Appendix A.2.

Recall that by definition a strongly uniform frame is such that: $\sum_{j=1}^{N}\left|H_{i j}(\omega)\right|^{2}=1$, $i=1, \ldots M$, for all $\omega$. Thus, as a consequence of the previous theorem we can state:

Corollary 1 Any oversampled filter bank which implements a strongly uniform tight frame is robust to one erasure.

The result of Theorem 3 does not reveal anything about the existence of filter banks which are robust to more than one erasure.

In [8], it has been shown that a complex harmonic frame in $\mathcal{C}^{N}$ or a real harmonic frame in $\mathcal{R}^{N}$ is robust to $e$ erasures $(e \leq M-N)$. A complex harmonic tight frame is given by: 


$$
\varphi_{k i}=\frac{1}{\sqrt{N}} W_{M}^{(k-1)(i-1)}, \quad i=1,2, \ldots, N, \quad k=1,2, \ldots, M ;
$$

where $W_{M}=e^{j 2 \pi / M}$. (A real harmonic tight frame could be defined similarly). The following theorem guarantees the existence of at least one family of strongly uniform tight frames in $l_{2}(\mathcal{Z})$ which are robust to e erasures $(e \leq M-N)$ :

Theorem 4 Consider an oversampled filter bank with polyphase analysis matrix $\mathbf{G}(\omega)=$ $F \mathrm{U}(\omega)$, where $F$ is a complex harmonic frame in $\mathcal{C}^{N}$ or a real harmonic frame in $\mathcal{R}^{N}$ and $\mathrm{U}(\omega)$ is an $N \times N$ polyphase matrix nonsingular on the unit circle $(\operatorname{det}(\mathbf{U}(\omega)) \neq 0)$. This filter bank is robust to e erasures $(e \leq M-N)$.

Proof: See Appendix A.3.

If $\mathbf{U}(\omega)$ is a paraunitary matrix, the resulting oversampled filter bank $\mathbf{G}(\omega)=F \mathbf{U}(\omega)$ represents a strongly uniform tight frame robust to $e$ erasures $(e \leq M-N)$.

\subsection{Effect of Erasures on the MSE}

In the previous section, it has been shown that it is possible to design oversampled filter banks which are robust up to $M-N$ erasures. We assume such filter banks for the rest of the paper.

Now, we want to compute the effect of the erasures on the MSE. Call $\mathbf{H}(\omega)$ the polyphase matrix related to the original frame and $\mathbf{H}_{E}(\omega)$ the polyphase matrix after $e=|E|$ erasures. The reconstruction uses the dual polyphase matrix $\mathbf{H}_{E}^{\dagger}(\omega)$ and the quantization model is the one proposed in (3)-(4). Under these assumptions the mean square error is equivalent to the one determined in (5):

$$
M S E_{E}=\frac{\sigma^{2}}{N} \frac{1}{2 \pi} \sum_{i=1}^{N} \int_{-\pi}^{\pi} \frac{1}{\lambda_{i}\left(\mathbf{H}_{E}^{*}(\omega) \mathbf{H}_{E}(\omega)\right)} d \omega,
$$

where $\lambda_{i}\left(\mathbf{H}_{E}^{*}(\omega) \mathbf{H}_{E}(\omega)\right), \quad i=1, \ldots, N$, are the spectral eigenvalues of $\mathbf{H}_{E}^{*}(\omega) \mathbf{H}_{E}(\omega)$.

However, our target is to express the mean square error in terms of the original frame and to find properties that the original frame operator has to satisfy to minimize the distortion. Consider first a strongly uniform frame and $e=1$ :

Theorem 5 Consider encoding with a strongly uniform frame and decoding with linear reconstruction. The MSE averaged over all possible erasures of one channel is minimum if and only if the original frame is tight. Moreover a tight frame minimizes the maximum distortion caused by one erasure. The MSE is given by:

$$
M S E_{1}=\left(1+\frac{1}{M-N}\right) M S E_{0} .
$$

It is not possible to extend the result of this theorem to the case of more than one erasure. However, it is possible to compute the $M S E$ with $e>1$ when the original frame is strongly uniform and tight:

$$
M S E_{E}=\left(1+\frac{1}{2 \pi} \int_{-\pi}^{\pi} \sum_{i=1}^{e} \frac{\mu_{i}(\omega)}{M-N \mu_{i}(\omega)} d \omega\right) M S E_{0}
$$


where $\mu_{i}(\omega)$ are the spectral eigenvalues of $\mathbf{T}^{*}(\omega) \mathbf{T}(\omega)$ and $\mathbf{T}(\omega)$ is the $N \times e$ polyphase matrix with columns $\left\{H_{i}(\omega)\right\}_{i \in E}$.

Since (6) is similar to (5), and the spectral sum of the e eigenvalues of $\mathbf{T}(\omega)$ is constrained to be a constant, the minimum in (6) occurs when all the eigenvalues are equal and constant, which is true when $\mathbf{T}(\omega)$ is tight.

Unfortunately, we cannot guarantee that for any possible erasure event the matrix $\mathbf{T}(\omega)$ is tight.

\section{Conclusions}

This work has presented extensions of the problem of quantized frame expansions with erasures $[7,8]$ to the case of oversampled filter banks and infinite-length input sequences. It has been shown that oversampled filter banks associated with tight frames perform the best in this context. Moreover, we presented practical ways to design oversampled filter banks robust to one or more than one erasures.

The design of filters which are optimal in this communication context and which show other desirable properties such as good space-frequency localization is under investigation.

\section{A. Appendix}

\section{Proof of Theorem 1}

Given a strongly uniform tight frame represented by the polyphase matrix $\mathbf{G}(\omega)$, all the other polyphase matrices related to the same equivalent class are obtained as follows:

$$
\mathbf{H}(\omega)=\mathbf{\Sigma} \mathbf{G}(\omega) \mathbf{U}(\omega),
$$

where $\mathbf{U}(\omega)$ is an $N \times N$ paraunitary matrix, $\boldsymbol{\Sigma}=\operatorname{diag}\left(\sigma_{1}, \sigma_{2}, \ldots . \sigma_{M}\right)$ and $\sigma_{k}= \pm e^{-j l \omega}$ $l \in \mathcal{Z}$. This equivalence class preserves tightness, uniformity, and also strong uniformity. Thus, if $\mathbf{G}(\omega)$ is strongly uniform and tight, so is $\mathbf{H}(\omega)$.

Now, let $\mathbf{H}(\omega)$ be a polyphase matrix associated with a strongly uniform tight frame with $M=N+1$. It can be shown that it consists of the first $N$ columns of a scaled $M \times M$ paraunitary matrix $\overline{\mathbf{H}}(\omega)$. Each row (or column) of $\overline{\mathbf{H}}(\omega)$ is of norm $\sqrt{M / N}$ that is:

$$
\sum_{j=1}^{N+1}\left|\bar{H}_{i, j}(\omega)\right|^{2}=\frac{N+1}{N} \quad i=1,2, . ., N+1 .
$$

Moreover, since our frame is strongly uniform we have:

$$
\sum_{j=1}^{N}\left|\bar{H}_{i, j}(\omega)\right|^{2}=1 \quad i=1,2, \ldots, N+1
$$

Subtracting (9) from (8) we obtain:

$$
\left|\bar{H}_{i, N+1}(\omega)\right|^{2}=\frac{1}{N} .
$$


Since $\mathbf{H}(\omega)$ is realized with FIR filters, it is formed only of Laurent polynomial elements. This implies that $\bar{H}_{i, N+1}(\omega)$ must be a monomial : $\bar{H}_{i, N+1}(\omega)= \pm N^{-1 / 2} e^{-j k \omega}$ $k \in \mathcal{Z}$. Without loss of generality we assume that $\bar{H}_{i, N+1}(\omega)= \pm N^{-1 / 2}$. That is, the last column of $\mathbf{H}(\omega)$ is $\left( \pm N^{-1 / 2}, \pm N^{-1 / 2}, \ldots, \pm N^{-1 / 2}\right)$ for some choice of signs.

Any given choice of signs in $\bar{H}_{i, N+1}(\omega)$ determines a subspace. Thus the span of the other $N$ subspaces (each subspace is related to one of the channels) must be the orthogonal complement to this subspace. Since orthonormal bases for a subspace are unitarily equivalent, the possible tight frames corresponding to a single choice of signs are in the same equivalence class. Flipping signs yields frames in the same equivalence class.

\section{Proof of Theorem 3}

Assume that the erased channel is $H_{i}(\omega)$. Call $\mathbf{H}_{i}(\omega)$ the polyphase matrix after one erasure. Then:

$$
\mathbf{H}_{i}^{*}(\omega) \mathbf{H}_{i}(\omega)=\underbrace{\mathbf{H}^{*}(\omega) \mathbf{H}(\omega)}_{\frac{M}{N} I_{N}}-H_{i}(\omega) H_{i}^{*}(\omega) .
$$

$\mathbf{H}_{i}(\omega)$ is a frame if and only if $\mathbf{H}_{i}^{*}(\omega) \mathbf{H}_{i}(\omega)$ is of full rank on the unit circle. That means that $\left(\mathbf{H}_{i}^{*}(\omega) \mathbf{H}_{i}(\omega)\right)^{-1}$ must exist on the unit circle. The identity:

$$
(A-B C D)^{-1}=A^{-1}+A^{-1} B\left(C^{-1}-D A^{-1} B\right)^{-1} D A^{-1}
$$

with $A=\frac{M}{N} I_{N}, B=H_{i}(\omega), C=1$, and $D=H_{i}^{*}(\omega)$ yields:

$$
\begin{aligned}
& \left(\mathbf{H}_{i}^{*}(\omega) \mathbf{H}_{i}(\omega)\right)^{-1}=\frac{N}{M} I_{N}+\frac{N}{M} I_{N} H_{i}(\omega)\left(1-H_{i}^{*}(\omega) \frac{N}{M} I_{N} H_{i}(\omega)\right)^{-1} H_{i}^{*}(\omega) \frac{N}{M} I_{N} \\
& =\frac{N}{M} I_{N}+\frac{N^{2}}{M^{2}}\left(1-\frac{N}{M} H_{i}^{*}(\omega) H_{i}(\omega)\right)^{-1} H_{i}(\omega) H_{i}^{*}(\omega) .
\end{aligned}
$$

Thus, the matrix is invertible if and only if:

$$
1-\frac{N}{M} H_{i}^{*}(\omega) H_{i}(\omega) \neq 0 \quad \text { for all } \omega .
$$

The desired inequality now follows from the fact that the frequency response of each filter is continuous (since we are only considering FIR filters) and the frame is uniform. The continuity of the filters implies that $\sum_{j=1}^{N}\left|H_{i j}(\omega)\right|^{2}<\frac{M}{N}$, for all $\omega$ or $\sum_{j=1}^{N}\left|H_{i j}(\omega)\right|^{2}>\frac{M}{N}$, for all $\omega$, but since the frames considered are uniform it must be that $\sum_{j=1}^{N}\left|H_{i j}(\omega)\right|^{2}<\frac{M}{N}$, for all $\omega$.

\section{Proof of Theorem 4}

First note that if a finite set of channels has a subset that is a frame, then the original set of channels is also a frame. Thus it suffices to consider subsets with $N$ channels; since all of these will be shown to be frames, larger subsets are also frames.

Let us call $\mathbf{G}_{E}(\omega)$ the $N \times N$ polyphase matrix after $e$ erasures $(e=M-N)$. $\mathbf{G}_{E}(\omega)$ is a frame if and only if $\operatorname{det}\left(\mathbf{G}_{E}(\omega)\right) \neq 0$ on the unit circle. Now, we know that $\operatorname{det}\left(F_{E}\right) \neq 0$ for any subset of $e=(M-N)$ erasures [8] and since: $\mathbf{G}_{E}(\omega)=F_{E} \mathbf{U}(\omega)$,

$$
\operatorname{det}\left(\mathbf{G}_{E}(\omega)\right)=\operatorname{det}\left(F_{E}\right) \operatorname{det}(\mathbf{U}(\omega)) \neq 0 \text { for all } \omega .
$$




\section{References}

[1] J.J. Benedetto and D. Colella, "Wavelet analysis of spectrogram seizure chirps", in Proc. SPIE Wavelet Appl. in Signal and Image Proc. III, vol. 2569, San Diego, CA, July 1995, pp. 512-521.

[2] J.J. Benedetto and G.E. Pfander, "Wavelet periodicity detection algorithms", in Proc. SPIE Wavelet Appl. in Signal and Image Proc. VI, vol. 3459, San Diego, CA, July 1998, pp.48-55.

[3] Z. Cvetkovic and M. Vetterli, "Oversampled Filter Banks" IEEE Trans. on Signal Proc., 46(5):1245:1255,1998

[4] I. Daubechies, Ten Lectures on Wavelets, Society for Industrial and Applied Mathematics, Philadelphia, PA, 1992.

[5] P.L. Dragotti, S.D. Servetto and M. Vetterli, "Analysis of Optimal Filter Banks for Multiple Description Coding," in Proc. IEEE Data Compression Conf., Snowbird, Utah, March 2000, pp. 323-332.

[6] V.K. Goyal, M. Vetterli and N. T. Thao, "Quantized overcomplete expansions in $\mathcal{R}^{N}$ : Analysis, synthesis and algorithms," IEEE Trans. Inform. Theory, 44(1):16-31, 1998.

[7] V.K. Goyal, J. Kovačević and M. Vetterli, "Quantized frame expansions as source-channel codes for erasure channels", in Proc. IEEE Data Compression Conf., Snowbird, Utah, March 1999, pp. 326-335.

[8] V.K. Goyal and J. Kovačević, "Quantized frame expansions with erasures", submitted to Applied and Computational Harmonic Analysis, to appear 2001.

[9] S. Mehrotra "Optimal overcomplete subband expansions for multiple description quantization," Presented at SPIE Conference, San Diego, CA, August 2000 .

[10] P.P. Vaidyanathan Multirate Systems and Filter Banks. Englewood Cliffs, NJ: Prentice-Hall, 1993.

[11] M. Vetterli and J. Kovačević, Wavelets and Subband Coding, Prentice Hall, 1995.

[12] X. Yang and K.Ramchandran "Optimal Subband Filter Banks for Multiple Description Coding", IEEE Trans. Inform. Theory, 46(7): 2477-2490, November 2000 . 